\title{
Multiwaveband analysis of brightest GRB070125
}

\author{
Poonam Chandra*, S. Bradley Cenko ${ }^{\dagger}$, Dale A. Frail ${ }^{* *}$, Roger A. \\ Chevalier $^{\star}$, Jean-Pierre Macquart ${ }^{\S}$, Shri R. Kulkarni ${ }^{\uparrow}$, Douglas C.-J. Bock ${ }^{\|}$ \\ and Frank Bertoldi ${ }^{\dagger}$ \\ ${ }^{*} J a n s k y$ Fellow, NRAO and University of Virginia, Charlottesville, VA 22904 \\ ${ }_{\dagger}^{\dagger}$ Space Radiation Laboratory, MS 220-47, California Institute of Technology, Pasadena, CA 91125 \\ ${ }^{*+}$ National Radio Astronomy Observatory, P.O. Box O, Socorro, NM 87801 \\ ¿Department of Astronomy, University of Virginia, Charlottesville, VA 22904 \\ $\S$ Jansky Fellow, NRAO and Div. of Physics, Maths and Astronomy, CalTech, Pasadena, CA 91125 \\ "Div. of Physics, Maths and Astronomy, California Institute of Technology, Pasadena, CA 91125 \\ "Combined Array for Research in Millimeter-wave Astronomy, P.O. Box 968, Big Pine, CA 93513 \\ ${ }^{\top}$ Argelander-Institut für Astronomie, Auf dem Hügel 71, D-53121 Bonn, Germany
}

\begin{abstract}
We present a comprehensive multiwavelength analysis of the bright, long duration gamma-ray burst GRB 070125, comprised of observations in $\gamma$-ray, X-ray, optical, millimeter and centimeter wavebands. Radio light curves show rapid flux variations, which are interpreted as due to interstellar scintillation, and are used to derive an upper limit of $2.4 \times 10^{17} \mathrm{~cm}$ on the radius of the fireball. Radio light curves and spectra suggest that the afterglow shock wave is moving in a dense medium. Our broadband modeling favors a constant density profile for the circumburst medium over a wind-like profile $\left(R^{-2}\right)$. GRB 070125 is a burst with high radiative efficiency (>60\%).
\end{abstract}

Keywords: gamma rays: bursts, radiation mechanisms: non-thermal, circumstellar matter PACS: 98.70.Rz. 96.60.tk, 41.60.Ap, 96.60.tg, 95.85.-e

\section{INTRODUCTION}

GRB 070125 was discovered by the Inter Planetary Network (IPN) of GRB detectors at 07:20:45 UT 25 January 2007 [1]. The Swift BAT detected the burst four minutes after the event. which enabled follow-up observations to identify the bright X-ray and optical afterglow. In this paper, we present multiwavelength observations of GRB 070125 . Its bright afterglow has allowed us to follow the GRB until day 350 and obtain the most extensive radio data in the Swift era, coupled with well-sampled X-ray and optical light curves indicative of a jet break. Together with the well characterized prompt emission extending beyond $1 \mathrm{MeV}$ [2], GRB 070125 is truly a rare event.

\section{OBSERVATIONS}

\section{X-ray and optical observations}

The X-ray Telescope (XRT; Burrows et al. 3) on board Swift began observing the field of GRB $07012546 \mathrm{ks}$ after the burst trigger [4]. The X-ray spectrum is well-fit by a power-law model with a photon index $\Gamma=2.1 \pm 0.3$ [4]. The XRT continued to monitor the X-ray afterglow of GRB 070125 over the course of the next two weeks. We obtained

CP1000, Gamma-Ray Bursts 2007: Proceedings of the Santa Fe Conference, edited by M. Galassi, D. Palmer, and E. Fenimore (c) 2008 American Institute of Physics 978-0-7354-0533-2/08/\$23.00 
time on the Chandra X-ray Observatory to observe the X-ray afterglow of GRB 070125 at very late times, which resulted in an upper limit.

In response to the IPN-Swift localization, we began observing the field of GRB 070125 with the automated Palomar 60-inch telescope (P60; Cenko et al. 5) on the night of 2007 January 26. Inspection of the first images revealed a bright, stationary source at $\alpha=0751$ 17.75, $\delta=+310904.2$ (J2000.0) [6], not present in the Sloan Digital Sky Survey (SDSS) images of the field [7]. We continued to monitor the afterglow of GRB 070125 with the P60 in the Kron $R$ and Sloan $i^{\prime}$ filters for the following four nights, until the afterglow faded below our sensitivity limit.

\section{Centimeter and sub-millimeter band observations}

We obtained director's discretionary time for observations in the 1.2-mm band ( 250 $\mathrm{GHz}$ ) at the Max-Planck Millimeter Bolometer Array (MAMBO), installed at the IRAM $30 \mathrm{~m}$ telescope on Pico Veleta, Spain. Our observations took place on 2007 January 30 and we detected the afterglow of GRB 070125 at a flux density of $3.14 \pm 0.59 \mathrm{mJy}$. We monitored the afterglow regularly until it had dropped below the instrumental sensitivity. Observations were also obtained on four occasions at $95 \mathrm{GHz}$ using the Combined Array for Research in Millimeter-wave Astronomy (CARMA). The GRB was detected twice.

The earliest measurement of the radio flux density was taken from the Westerbork Synthesis Radio Telescope (WSRT) in $5 \mathrm{GHz}$ band by van der Horst [8]), just 1.5 days after the burst. A $2-\sigma$ upper limit on the $5 \mathrm{GHz}$ flux density of $F_{v}<174 \mu \mathrm{Jy}$ was obtained. Shortly thereafter, we triggered Very Large Array (VLA) observations of the field. Our first measurement at $8.46 \mathrm{GHz}$, four days after the explosion, resulted in a strong detection $\left[F_{V}=360 \pm 42 \mu \mathrm{Jy} ; 9\right]$. We followed the GRB until day 342 since explosion in all VLA bands.

\section{RESULTS}

\section{Scintillation}

We obtained long-duration observations of the afterglow of GRB 070125 at $8.5 \mathrm{GHz}$ on three separate occasions: February 7 ( 5.5 hour duration), February 8 (4 hour duration), and February 14 ( 8 hour duration). The data were split into 20 minute blocks and imaged in order to extract information on the fast variability of the source. GRB 070125 exhibits flux density variations with a significance exceeding $99.8 \%$ on each of the three epochs. While the short baseline hindered a definitive determination, we tentatively identify breaks at $\Delta T \approx 6 \times 10^{3}, 7 \times 10^{3}$ and $9 \times 10^{3} \mathrm{~s}$ at the three occasions, respectively.

The interpretation of the variability depends on whether the scintillation occurs in the weak or strong regime. Strong scintillations require that the so-called Fried parameter (coherence length scale, $\left.s_{d}\right)$ be smaller than the Fresnel size $\left(r_{F}\right)$, and is possible only for sources smaller than $\lambda / s_{d}$. These lead to the following two conditions [10]:

$$
v<13.4\left(\mathrm{SM} / 10^{-3.19} \mathrm{~m}^{-20 / 3} \mathrm{kpc}\right)^{6 / 17}\left(D_{\mathrm{scr}} / \mathrm{kpc}\right)^{5 / 17} \mathrm{GHz} \equiv v_{\mathrm{ss}}
$$




$$
\theta_{d}=6.5(v / 8.46 \mathrm{GHz})^{-11 / 5}\left(\mathrm{SM} / 10^{-3.19} \mathrm{~m}^{-20 / 3} \mathrm{kpc}\right)^{3 / 5} \mu \text { as } \ll \sqrt{\mathrm{c} / 2 \pi v \mathrm{D}_{\text {scr }}} \text {, }
$$

where SM is the scattering measure and $D_{\mathrm{scr}}$ is the effective distance to the scattering material. To determine this, we first estimate the scattering distance and the scattering measure using the formulation of Cordes and Lazio [11]. Given the Galactic coordinates of GRB $070125,(l, b)=(189.4,25.6)$, the expected SM is $10^{-3.19} \mathrm{~m}^{-20 / 3} \mathrm{kpc}$ and the effective distance to the scattering material is $D_{\text {scr }}=0.84 \mathrm{kpc}$. For these parameters, the critical frequency obtained from Eq. 1 is $12.7 \mathrm{GHz}$. These parameters also satisfy the condition of Eq. 2. However, Strong scintillation can be diffractive as well refractive in nature. The size limit for a source to exhibit diffractive scintillation $\left(\theta_{1}\right)$ is [10]:

$$
\theta_{1}=1.2(v / 8.46 \mathrm{GHz})^{6 / 5}\left(D_{\mathrm{scr}} / \mathrm{kpc}\right)^{-1}\left(\mathrm{SM} / 10^{-3.19} \mathrm{~m}^{-20 / 3} \mathrm{kpc}\right)^{-3 / 5} \mu \text { as. }
$$

For actual size of the source, $\theta_{s}, \theta_{s} \leq \theta_{1}$. Diffractive scintillation occurs when $\theta_{d}>\theta_{1}$, which is indeed the case for GRB 070125 using the parameters discussed above. This equation gives upper limit on the radius of the source as $\leq 2.4 \times 10^{17} \mathrm{~cm}$.

\section{Broadband modeling}

Here we combine all the observations of GRB 070125 in an attempt to derive a comprehensive model of the entire afterglow evolution. Full details of the model can be found in Yost [12] and Yost et al. [13]. We ignore all data before $t=1 \mathrm{~d}$ due to the possibility of late-time energy injection. We fit the data using both wind like as well as constant density profiles. In terms of $\chi^{2}$ and the model-fit statistic, the wind model does a slightly better job than the ISM model. However, the resulting best-fit parameters for the wind model are largely unphysical. The extremely small isotropic afterglow kinetic energy $\left(E_{52} \approx 0.3\right)$ compared to the $\gamma$-ray isotropic energy is also troubling. The magnetic field fraction required reaches $100 \%$ in this model.

The ISM model gives values of various parameters closer to the ones obtained from our simple analytical models. The isotropic kinetic energy $\left(\sim 8 \times 10^{52}\right.$ erg)is much smaller than the isotropic gamma-ray energy obtained from the Konus-Wind/RHESSI fluence $\left(10^{54} \mathrm{erg}\right)$. This may indicate that either there are high radiative losses at early times or the prompt emission is rather efficient with an extremely high value of $\eta_{\gamma}$.

In Figure 1, we plot the broadband spectra from radio to X-ray at various times of the afterglow evolution. They are represented well with our models. Both the wind and ISM environments seem to do an equally good job.

\section{CONCLUSION}

GRB 070125 is one of the brightest GRBs ever detected, both in terms of its prompt high-energy fluence and its optical and radio afterglows. This is the most extensively followed GRB in multiwavebands in the Swift era. The richness of the data allowed us 

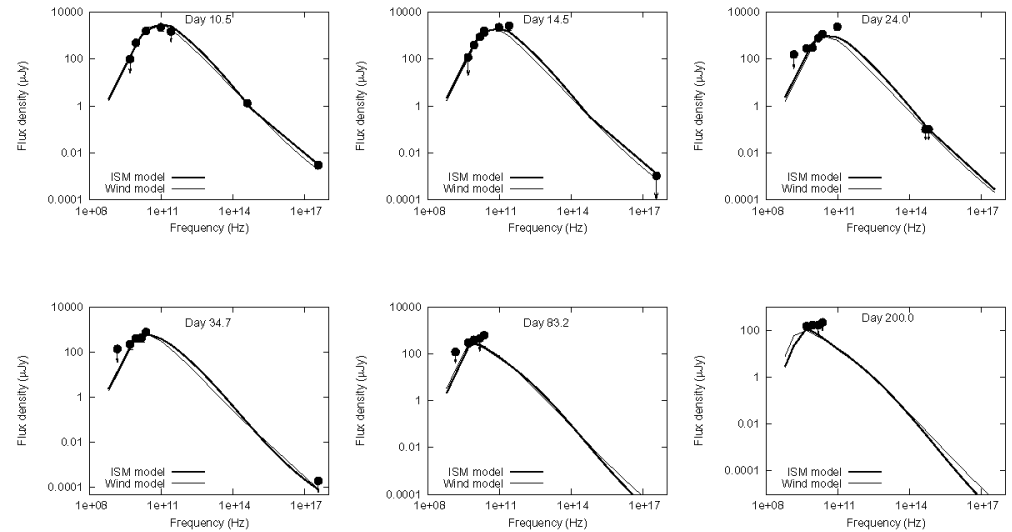

FIGURE 1. Broad band spectra at various days. The thick solid line is the ISM model and the thin solid line represents the Wind model. The peak flux density is well constrained due to submm observations.

to derive many important properties of the GRB and place useful constraints on many parameters. The radio data gave evidence for diffractive scintillations, which gave an upper limit on the size of the fireball. This estimate of the fireball size is consistent with the one obtained from the broadband modeling in a constant density medium. The parameter values in the ISM model are more robust and change little with little change in the input values, unlike the wind model which is rather unstable. In both the ISM and the wind models, the radiative efficiency of the GRB is very high $(>60 \%)$.

\section{ACKNOWLEDGMENTS}

PC thanks the VLA staff for making radio observations. We thank Sarah Yost for providing us the GRB broadband modeling code and helping out in running the code. RAC was supported in part by NASA grant NNG06GJ33G.

\section{REFERENCES}

1. K. Hurley, et al., GRB Coordinates Network 6024, 1-+ (2007).

2. E. C. Bellm, et al., ArXiv e-prints 710 (2007), 0710.4590.

3. D. N. Burrows, , et al., Space Science Reviews 120, 165-195 (2005).

4. J. Racusin, and L. Vetere, GRB Coordinates Network 6030, 1-+ (2007).

5. S. B. Cenko, et al., PASP 118, 1396-1406 (2006), astro-ph/ 0608323.

6. S. B. Cenko, and D. B. Fox, GRB Coordinates Network 6028, 1-+ (2007).

7. J. K. Adelman-McCarthy, et al., SDSS (2007), arXiv:astro-ph/0707.3413, 0707.3413.

8. A. J. van der Horst, GRB Coordinates Network 6042, 1-+ (2007).

9. P. Chandra, and D. A. Frail, GRB Coordinates Network 6061, 1-+ (2007).

10. J. Goodman, New Astronomy 2, 449-460 (1997), arXiv : astro-ph/9706084.

11. J. M. Cordes, and T. J. W. Lazio, ArXiv Astrophysics e-prints (2002), astro-ph/ 0207156.

12. S. A. Yost, Gamma-ray burst afterglows, Ph.D. thesis, AA(CALTECH) (2004).

13. S. A. Yost, et al., ApJ 597, 459-473 (2003), arXiv: astro-ph/0307056. 